\title{
6th International Workshop on Foundations and Practices of UML (FP-UML 2010)
}

\section{Preface}

The Unified Modeling Language (UML) has been widely accepted as the standard object-oriented (OO) modeling language for modeling various aspects of software and information systems. The UML is an extensible language, in the sense that it provides mechanisms to introduce new elements for specific domains if necessary, such as web applications, database applications, business modeling, software development processes and data warehouses. Furthermore, the latest version of UML 2.0 got even bigger and more complicated with more diagrams for some good reasons. Although UML provides different diagrams for modeling different aspects of a software system, not all of them need to be applied in most cases. Therefore, heuristics, design guidelines, lessons learned from experiences are extremely important for the effective use of UML 2.0 and to avoid unnecessary complication. Also, approaches are needed to better manage UML 2.0 and its extensions so they do not become too complex to manage in the end.

The Sixth International Workshop on Foundations and Practices of UML (FPUML'10) intends to be a sequel to the successful BP-UML'05 - FP-UML'09 workshops held in conjunction with the ER'05 - ER'09, respectively. FP-UML'10 intends to be a premier forum for exchanging ideas on the best and new practices of the use of UML in modeling and system development. As Booch et al. have stated, "the full value of model driven architecture is only achieved when the modeling concepts map directly to domain concepts rather than computer technology concepts", domain specific approaches to UML were particularly encouraged.

We received 10 full papers. The Program Committee only selected 5 papers, making an acceptance rate of $50 \%$. The accepted papers were organized in two sessions. The first one will be focused on Semantics and Ontologies in UML, where the first two papers deal with using UML for enterprise and service oriented architecture modeling, and the third tackles the meaning of membership in collections. In the second session, two papers focusing on Automation and Transformation of activities in UML will be presented.

We hope that you will enjoy this record of the workshop and find the information within these proceedings valuable towards your understanding of the current state-ofthe-art in UML modeling issues. We would like to express our gratitude to the program committee members for their hard work in reviewing papers, the authors for submitting their papers, and the ER 2010 organizing committee for all their support. 